\title{
Intervertebral foramen injection of plerixafor attenuates CCD-induced low back pain in rats through down-regulation of Nav1.8 and Nav1.9
}

\section{Fei Yang}

Clinical Medical College (900 Hospital of the Joint Logistic Support Force), Fujian Medical University, Fuzhou 350025, Fujian, P.R. China

\section{Huang-Hui Wu}

Dongfang Hospital, Xiamen University

\section{Lu Chen}

Department of Anesthesiology, The First Affliated Hospital, Fujian Medical University, Fuzhou 350005, Fujian, P.R. China

\section{Guo-Zhong Chen}

Department of Anesthesiology and Perioperative Medicine, Clinical Medical College ( 900 Hospital of the Joint Logistic Support Force), Fujian Medical University, Fuzhou 350025, Fujian, P.R. China. Department of Anesthesiology and Perioperative Medicine, Don

\section{Xiao-Zhi Wu ( $\nabla$ www7410@sohu.com )}

Department of Anesthesiology and Perioperative Medicine, Clinical Medical College (900 Hospital of the Joint Logistic Support Force), Fujian Medical University, Fuzhou 350025, Fujian, P.R. China. Department of Anesthesiology and Perioperative Medicine, Don

\section{Research}

Keywords: low back pain, dorsal root ganglion, CXCR4, plerixafor, intervertebral foramen, Nav1.8

Posted Date: October 6th, 2020

DOI: https://doi.org/10.21203/rs.3.rs-76978/v1

License: (a) (i) This work is licensed under a Creative Commons Attribution 4.0 International License. Read Full License 


\section{Abstract}

\section{Background}

Low back pain, an extremely common chronic pain, is now the number one cause of disability globally. On the chronic compression of the dorsal root ganglion (CCD) model, a typical low back pain model, previous study has discovered that C-X-C chemokine receptor type 4 (CXCR4) involved in mediating low back pain. However, the underlying molecular mechanisms and whether it has the potential to serve as an applicable target for clinical treatment of low back pain remains unclear.

\section{Methods}

CCD-induced low back pain model was established by inserting stainless steel L-shape rod into the intervertebral foramen at L5 level. Multiple pain behaviors including mechanical hyperalgesia and allodynia, thermal hyperalgesia and cold allodynia were evaluated. The role of CXCR4 in CCD-induced pain was assessed by intraganglionar CXCR4 siRNA injection and intervertebral foramen injection of plerixafor, a selective CXCR4 antagonist. The expression change of CXCR4, Nav1.8 and Nav1.9 was examined by immunofluorescent staining.

\section{Results}

We showed that the CXCR4 were dramatically up-regulated in the compressed dorsal root ganglion (DRG) neurons in CCD model, and intraganglionar CXCR4 siRNA injection significantly reduced CCD-induced multiple pain behaviors, including mechanical hyperalgesia and allodynia, thermal hyperalgesia and cold allodynia. The expression of Nav1.8 and Nav1.9 in the compressed DRG were also enhanced after CCD, and which were remarkably reversed by intervertebral foramen injection of plerixafor at the compression level. Moreover, intervertebral foramen injection at the compression level of plerixafor and ropivacaine as well as A-803467, a Nav1.8 selective blocker, were all able to reverse CCD-induced multiple pain behaviors. However, the analgesic duration of plerixafor maintained at least for $24 \mathrm{~h}$ which was much longer than that of A-803467 and ropivacaine. Finally, we found intervertebral foramen injection of plerixafor at the adjacent non-compression level has no effect on CCD-induced pain and intervertebral foramen injection of plerixafor in normal rats did not affect their basal pain sensitivity.

\section{Conclusions}

Our study provides evidence that CXCR4-Nav1.8/Nav1.9 axis in compressed DRG contributed to CCDinduced low back pain and intervertebral foramen injection of plerixafor was a potential and applicable therapeutic strategy for the treatment of low back pain.

\section{Background}

Low back pain, the most common chronic pain, is the leading cause of disability and higher health care costs over the world which severely affect the patients' quality of daily life [1]. For many patients, the 
pharmacotherapy of low back pain is challenging and the effective treatment methods is lacking [2]. Currently, an urgent need exists for the development of novel, more effective treatment approaches and drugs for low back pain. To narrow the gap between understanding the underlying mechanism and its clinical treatment, a chronic compression of dorsal root ganglion (CCD)-induced low back pain model in murine has been developed attempting to mimic the common clinical causes of low back pain, such as intervertebral disc laterally herniation, intervertebral foramen stenosis, or other disorders affecting the dorsal root ganglion (DRG) or its near nerve root [3-5]. More and more work is endeavoring to explore the new potential targets or strategy for pain relief on CCD model.

The chemokine CXC motif receptor 4 (CXCR4) belonging to the well-studied G protein-coupled receptor (GPCR) family is a primary cognate receptor for the chemokine CXC motif ligand 12 (CXCL12) which plays an important role in many biological functions, including embryogenesis, immune surveillance, inflammation response, tissue homeostasis, tumor growth and metastasis [6-8]. Much evidence has accumulated that CXCR4 chemokine signaling contributes to the development and maintenance of chronic pain [9-11]. A recent study have demonstrated that intraperitoneal injection of plerixafor (AMD3100), a selective antagonist for CXCR4, which has been approved for mobilization of hematopoietic stem cells clinically by the U.S. Food and Drug Administration in 2008, significantly ameliorated CCD-induced mechanical and thermal hyperalgesia, suggesting CXCR4 might be an adequate analgesic target [12-14]. As we known, the CXCR4 is widely located in the nervous system and distributed in various kinds of cells $[9,15]$. Consequently, the abovementioned drug systemic administration modality will inevitably result in a number of adverse events and blur the action site of the plerixafor. Giving that the dorsal root ganglion (DRG) containing bodies of primary sensory neurons which may generate abnormal spontaneous discharges and become an important source of hyperexcitability and increased nociceptive signaling, the intervertebral foramen injection aiming at delivering drugs onto the DRG might be an appropriate alternative approach for drug delivery [16-18].

Emerging data verified that the CCD causes an increase in the excitability of the cell bodies of compressed DRG neurons and the CCD-induced spontaneous activity and/or lowered rheobase of DRG neurons was accompanied, and possibly caused by, an increased expression of voltage-gated sodium currents (VGSCs) [19-21]. Recent research proved that knockdown of NaV1.6 and regulatory NaVß4 sodium channel markedly reduced spontaneous pain and mechanical and cold hyperalgesia induced by CCD, indicating that targeting the VGSCs may have therapeutic value for low back pain [22]. However, the roles of two other distinct VGSCs identified in DRG neurons, Nav1.8 which plays an important role in action potential generation, and Nav1.9 which contribute to setting the resting membrane potential and to neuronal excitability [23], have not yet been explored in CCD-induced low back pain. Our previous study has showed that activation of CXCR4 could directly modulate primary neuronal excitability by mediating the up-regulation of Nav1.8 under inflammatory pain state [24]. Interestingly, the CXCR4 expression also significantly increased in the compressed DRG from CCD mice [12]. Whether the Nav1.8 and Nav1.9 was the downstream effector target for CCD-induced CXCR4 activation remains unclear. 
Considering all, we set out to investigated whether CXCR4 in the compressed DRG participated in CCDinduced multiple pain behaviors, and then tested whether delivery of plerixafor to the compressed DRG by intervertebral foramen injection was an effective approach to management CCD-induced pain. Moreover, we extended our study to demonstrate that CXCR4 activation regulated the expression of Nav1.8 and Nav1.9. The present findings might provide preclinical evidence for targeting CXCR4 by intervertebral foramen injection as a potential therapeutic strategy for the treatment of low back pain.

\section{Materials And Methods}

\subsection{Animals}

Experiments were performed on male Sprague-Dawley rats (200-250 g, provided by Laboratory Animal Center of Fuzhou General Hospital). The animals were housed in plastic cage under specific pathogenfree conditions with a $12 \mathrm{~h}$ light/dark cycle and with free access to water and food. This study was carried out in accordance with the recommendations of the Animal Care and Use Committee of Fuzhou General Hospital and performed in accordance with the updated Guide for the Care and Use of Laboratory Animals. The number of rats used and their suffering were minimized. During the whole experiment, the rats were randomized

\subsection{Chronic compression of DRG}

CCD model were established according to the methods described previously [25]. Under anesthesia of sodium pentobarbital ( $50 \mathrm{mg} / \mathrm{kg}$, i.p.), the rats were fixed in a prone position and the left transverse process and intervertebral foramina of L5 were exposed. A stainless steel L-shape rod was implanted unilaterally into the intervertebral foramen L5. After the rod was implanted, the wound was closed by suturing muscle and skin layers. The surgical procedure of sham surgery was identical to that described above but without the rod insertion. All rats were allowed to recover in cages from anesthesia and surgery with food and water available at libitum. These rats with autophagy phenomenon, feeling deficiency, and disability were eliminated.

\subsection{Intraganglionar siRNA microinjection}

Intraganglionar siRNA microinjection was just prior to the insertion of metal L-shape rod. By using in vivo transfection reagent ( R0541, Thermo Scientific Inc) as the delivery vehicle, the CXCR4 small interfering RNA (siRNA) solution ( $2 \mu \mathrm{l}, 10 \mu \mathrm{M}$, sc-270577, Santa Cruz) or no-targeting control scrambled siRNA ( $2 \mu \mathrm{l}$, $10 \mu \mathrm{M}, \mathrm{sc}-37007$, Santa Cruz) was injected close to L5 DRG with a glass Hamilton microsyringe. The microsyringe was remained for 5 min after injection to prevent spread of the agent followed by rod implanted into the intervertebral foramen L5.

\subsection{Intervertebral foramen injection}


Intervertebral foramen drug injection was implemented as we previously described ${ }^{16}$. Briefly, rats were anesthetized with sevoflurane and the fur over the lower back were shaving. A $30 \mathrm{G}$ injecting needle was inserted towards the L5 intervertebral foramen or L4 intervertebral foramen through the skin at an angle of $45^{\circ}$ both to the horizontal and sagittal plane until the tip touched the lateral region of the vertebrae. To localize the L5 or L4 intervertebral foramen, delicate movements of the needle were made until the bone resistance was diminished and a paw flinch reflex was observed. The paw flinch reflex was used as a sign that the needle tip enters in the intervertebral foramen. In all intervertebral foramen injection, $10 \mu \mathrm{L}$ of solution was injected over a period of 15 seconds and saline was served as the control for plerixafor (Abcam, 10, 50, $100 \mu \mathrm{g} / 10 \mu \mathrm{L}$ ), A803467 (Abcam, $30 \mu \mathrm{g} / 10 \mu \mathrm{L}$ ) and ropivacaine (AstraZeneca LP, 40 $\mu \mathrm{g} / 10 \mu \mathrm{L}$, equivalent to $0.4 \%$ in concentration). To confirm the intervertebral foramen injection, the presence or absence of dye (Dil, 2.5\%) was visually confirmed by the exposure of the lumber region after each experiment was completed

\subsection{Mechanical pain sensitivity testing}

The mechanical pain sensitivity testing was performed by experimenters blinded to the experimental treatments. For examination of mechanical pain sensitivity, the mechanical stimuli were applied by using a series of von Frey monofilaments with different bending forces. The rats were placed on a metal mesh floor covered with plastic box, and von Frey filaments were applied from underneath the metal mesh floor to the plantar area of the hind paws. Each von Frey filament was applied five times (once every several seconds) in order to induce the withdrawal reflex. The bending force value of the von Frey filament that caused an appropriate $50 \%$ occurrence of paw withdrawal was expressed as the paw withdrawal mechanical threshold (PWMT, g).

\subsection{Thermal pain sensitivity testing}

The thermal sensitivity was tested by a blinded researcher and determined by measuring the withdrawal latency of the hind paws in response to radiant heat. Rats were placed in a plastic chamber on the surface of a 2-mm thick glass plate and the sensitivity of hindpaw to thermal stimuli by a radiant heat stimulator was measured. The latency was determined as the duration from the beginning of heat stimuli to the occurrence of a marked withdrawal reflex. Five stimuli were repeated, and the latter three values were averaged as mean paw withdrawal thermal latency (PWTL, s). A maximal cutoff of $30 \mathrm{~s}$ was used to avoid excessive tissue injury. The inter-stimulus interval for each heat test was more than $10 \mathrm{~min}$.

\subsection{Mechanical allodynia testing}

The mechanical allodynia testing was performed by experimenters blinded to the experimental treatments For examination of mechanical allodynia, the rats were habituated on a metal mesh floor covered with transparent plastic box. A wisp of cotton pulled up from, but still attached to a cotton swab was stroked mediolaterally across the plantar surface of the hindpaws to measure the presence of a withdrawal reflex to a normally innocuous mechanical stimulus (light touch-evoked tactile allodynia) 
which does not evoke a response in normal animals. The percentage of withdrawal response to ten cotton stimuli were calculated.

\subsection{Cold allodynia testing}

The cold allodynia was tested by a blinded researcher and determined by measuring the withdrawal response of hindpaws to a drop of acetone applied to the ventral surface of the hind paw. Using a flat-top syringe, we applied a droplet of acetone to the ventral surface of the hind paw of each rat through metallic mesh floor and observed their withdrawal responses. Acetone was applied alternately ten times to each hindpaw and the total percentage of withdrawal response were calculated.

\subsection{Immunohistochemistry}

The rats were anesthetized with sodium pentobarbital (50 mg/kg, i.p.), then perfused with physiological saline, followed by $4 \%$ paraformaldehyde in $0.1 \mathrm{M}$ PB solution. After perfusion, the L5 DRGs were removed and postfixed in the same $4 \%$ fixative overnight at $4{ }^{\circ} \mathrm{C}$ and cryoprotected by immersion in $30 \%$ sucrose in $0.1 \mathrm{M} \mathrm{PB}$ at $4{ }^{\circ} \mathrm{C}$. Transverse frozen sections $(15 \mu \mathrm{m}$ thick) were cut on CM1900 freezing microtome (Leica, Germany).Sections were blocked with $10 \%$ goat serum in phosphate buffered saline (PBS) for $2 \mathrm{~h}$ at room temperature and incubated with rabbit anti-Nav1.8(Alomone, CAT\# ASC016, 1:200), rabbit anti-Nav1.9 (Alomone, CAT\# ASC017, 1:200) or goat anti-CXCR4 (Abacm, CAT\# ab1670, 1:200) at $4{ }^{\circ} \mathrm{C}$ overnight. After three washes with PBS, the sections were incubated with Cy3-conjugated anti-rabbit secondary antibodies (Sigma, CAT\#C2306, 1:200) or Cy3-conjugated anti-goat secondary antibodies (Sigma, CAT\#C2821, 1:200) for 2-3 $\mathrm{h}$ at room temperature. The images were examined under a laser scan confocal fluorescent microscope (Olympus FV1000, Japan) and the immunofluorescence intensity was analyzed by Image $\mathrm{J}$ software.

\subsection{Statistical analysis}

Data were analyzed using GraphPad Prism version 7.0 (GraphPad,San Diego, CA, USA) and all data were expressed as means \pm SD. Behavioral time course data were analyzed using a two-way repeated measures ANOVA with Holm-Sidak's post hoc test. Differences in immunofluorescence intensity of each group were tested using t-tests or one-way ANOVA followed by Tukey's posttest. A level of $P<0.05$ was accepted as significant.

\section{Results}

\subsection{Knocking down the up-regulation of CXCR4 in the compressed DRG reduced CCD-induced multiple pain behaviors}

Representative images of CXCR4 immunofluorescence staining in DRG sections from sham DRG and from compressed DRG on day 3 are shown in Fig.1A. The mean immunofluorescence intensity of CXCR4 in compressed DRGs were significantly increased comparing with that from sham DRGs (Fig.1B). 
To determine whether the up-regulation of CXCR4 was involved in the CCD-induced pain behaviors, we knocked down the expression of CXCR4 by using intraganglionar siRNA microinjection as we reported previously [26]. As shown in the Fig.2A and Fig.2B, intraganglionar CXCR4 siRNA microinjection remarkably reduced CXCR4 expression in compressed DRG comparing with scrambled siRNA injection group. Previous studies have shown that CCD induces obvious mechanical and thermal pain sensitivity in the ipsilateral hindpaw as measured by the von Frey test and thermal stimuli, mechanical allodynia as measured by the paw withdrawal responses to a light cotton wisp stroking and cold allodynia as measured by increased withdrawal to acetone stimuli [22]. These multiple pain behaviors were also occurred as early as 1 day post CCD and maintained at least for 14 days (Fig.2). We found knockdown of CXCR4 expression in compressed DRG by intraganglionar CXCR4 siRNA microinjection significantly attenuated CCD-induced mechanical and thermal pain sensitivity over the whole observation period (Fig.2C and D). Additionally, the mechanical and cold allodynia in CCD rats were also improved by intraganglionar CXCR4 siRNA microinjection (Fig.2E and F).

\subsection{Intervertebral foramenplerixafor injection reduced CCD-induced multiple pain behaviors}

We used a selective and effective CXCR4 inhibitor plerixafor to assess whether focal intervertebral foramen injection was a potential new and effective route in treatment of CCD-induced low back pain. Single plerixafor $(10,50$ or $100 \mu \mathrm{g})$ was intervertebral foramen injection at L5 ipsilaterally on day 3 post CCD and CCD-induced multiple pain behaviors were evaluated at $0.5,1,3,5,24$ and 48 hour after injection. As shown in Fig. 3A and Fig. 3B, intervertebral foramen plerixafor (50 and $100 \mu \mathrm{g}$ ) injection dose-dependently decreased CCD-induced mechanical and thermal pain sensitivity compared to saline injection, while low dose of plerixafor $(10 \mu \mathrm{g})$ has no significant effect on decreased PWMT and PWTL induced by CCD comparing with saline injection. The analgesic effect of plerixafor (50 and $100 \mu \mathrm{g}$ ) initiated at $1 \mathrm{~h}$, reaching peak at $5 \mathrm{~h}$ and lasting for $24 \mathrm{~h}$ after intervertebral foramen injection. The mechanical and cold allodynia induced by CCD were also attenuated by intervertebral foramen plerixafor (50 and $100 \mu \mathrm{g}$ ) injection (Fig.3C and D). The ipsilateral paw withdrawal responding to cotton wisp stroking and acetone stimuli significantly decreased starting at $1 \mathrm{~h}$ and lasting for $24 \mathrm{~h}$ after plerixafor $(50$ and $100 \mu \mathrm{g})$ injection. However, the plerixafor $(10 \mu \mathrm{g})$ did not significantly affect CCD-induced mechanical and cold allodynia over $48 \mathrm{~h}$ (Fig.3C and D).

\subsection{Intervertebral foramenplerixafor injection reduced CCD-induced up-regulation of Nav1.8 and Nav1.9}

It has been demonstrated that activation of CXCR4 increased the excitability of DRG neurons through a mechanism involving voltage gated sodium channels (VGSCs) [27]. We further confirmed that Nav1.8 and Nav1.9 was a downstream effector of CXCR4 by examining the effect of plerixafor on Nav1.8 and Nav1.9 expression. As shown in Fig.4A and Fig.4B, the expression level of Nav1.8 and Nav1.9 in compressed DRGs of CCD rats remarkably increased compared to that DRGs from sham rats. However, intervertebral foramen plerixafor injection significantly reversed CCD-induced the up-regulation of Nav1.8 and Nav1.9 expression. These results suggesting that Nav1.8 and Nav1.9 might mediate the pronociceptive effect of CXCR4 activation under CCD condition. 


\subsection{Intervertebral foramen A803467 and ropivacaine injection reduced CCD-induced multiple pain behaviors}

We next sought to explore the role of VGSCs in CCD-induced multiple pain behaviors by using a selective Nav1.8 blocker, A803467 and a non-selective local anesthetics, ropivacaine which was commonly used in clinic. As shown in Fig.5A and Fig.5B, CCD-induced mechanical and thermal hyperalgesia were significantly reversed by ipsilaterally intervertebral foramen A803467 (30 $\mu \mathrm{g})$ injection at L5 level, and the analgesic effect of A803467 starting at $0.5 \mathrm{~h}$, reaching peak at $1 \mathrm{~h}$ and lasting for only $3 \mathrm{~h}$ after injection. The mechanical and cold allodynia induced by CCD were also attenuated by A803467 (30 $\mu \mathrm{g})$ from $0.5 \mathrm{~h}$ to $3 \mathrm{~h}$ after intervertebral foramen injection (Fig.5C and D), indicating that up-regulation of Nav1.8 in compressed DRG contributed to the maintenance of CCD-induced low back pain. Likewise, ropivacaine intervertebral foramen injection at L5 level could also remarkably reversed CCD-induced mechanical and thermal hyperalgesia, as well as the mechanical and cold allodynia (Fig.5). The analgesic effect of ropivacaine $(40 \mu \mathrm{g})$ was onset at $0.5 \mathrm{~h}$ and remained at the peak level for $5 \mathrm{~h}$, which was more significant than $\mathrm{A} 803467$ (Fig.5). However, at $24 \mathrm{~h}$ after intervertebral foramen injection, the anti-hyperalgesia and anti-allodynia effect of A803467 and ropivacaine both vanished (Fig.5).

\subsection{Intervertebral foramenplerixafor injection at L4 had no effect on CCD-induced multiple pain behaviors}

To address the question whether the analgesic effect of plerixafor attributed to its systemic effect, we performed plerixafor $(100 \mu \mathrm{g})$ injection at L4 intervertebral foramen. Over the whole time after L4 intervertebral foramen plerixafor injection, the PWMT and PWTL, as well as the withdrawal responding to cotton wisp stroking and acetone stimuli remained as they had been before plerixafor injection (Fig.6), indicating that the present dose of plerixafor intervertebral foramen injection has no systemic analgesic effect.

\subsection{Intervertebral foramenplerixafor injection had no effect on the basal mechanical and thermal pain sensitivity}

It has been reported that CXCR4 was constitutively expressed in primary afferent neurons and remain at a low expression level under physiological status while extremely increased under pathological condition. To evaluate the significance of this functional up-regulation of CXCR4, we applied L5 intervertebral foramen plerixafor injection in normal rats. As shown in Fig.7A, L5 intervertebral foramen plerixafor injection did not alter the ipsilateral PWMT to Von Frey test over the whole observed time. Also, there was no change in ipsilateral PWTL to thermal stimuli after plerixafor injection (Fig.7A). These results indicated that CXCR4 in DRG did not contribute to the basal pain sensitivity.

\section{Discussion}

CXCR4 is a recently reported chemokine receptor that plays a pivotal role in mediating neuropathic pain [9]. In the present study, we first demonstrated that CXCR4 expression significantly increased in compressed DRG from CCD rats. Knockdown of CXCR4 remarkably attenuated CCD-induced multiple 
pain behaviors, including mechanical and thermal hyperalgesia, mechanical and cold allodynia, suggesting that CXCR4 contributed CCD-induced low back pain. Moreover, we showed that intervertebral foramen injection of plerixafor, a CXCR4 selective antagonist, was able to reverse CCD-induced pain behaviors by reducing CCD-induced Nav1.8 and Nav1.9 up-regulation in compressed DRG. We further found that the Nav1.8 selective blocker, A-803467 and a non-selective VGSCs blocker, ropivacaine cold both relieve CCD-induced pain behaviors after intervertebral foramen injection, but the analgesic duration was apparently shorter than that of plerixafor. Finally, we demonstrated that intervertebral foramen injection of plerixafor eased CCD-induced pain due to its local effect on compressed DRG rather than the systemic effects, and the approach of intervertebral foramen plerixafor injection has no significant effect on the basal pain sensitivity. Taken together, these findings suggested that chemokine receptor CXCR4 involved in CCD-induced pain by up-regulating Nav1.8 and Nav1.9 expression, while intervertebral foramen plerixafor injection is a novel and appropriate therapeutic strategy to treat low back pain.

\subsection{CXCR4-Nav1.8/Nav1.9 axis contributes to CCD-induced multiple pain behaviors}

Low back pain is a major health problem over the world which is becoming worse largely because of the increase in the population base and the intensification of aging [28]. Despite the huge medical investment in the low back pain, its global burden is still heavy. During the past few decades, the clinical management of low back pain remains unsatisfactory. Commonly, low back pain closely related to the intraforaminal stenosis, laterally herniated disk, vertebral fracture, or inflammatory disorders [2]. To develop an effective treatment, it is important to understand the underlying mechanism of low back pain, which necessarily promoted a number of preclinical models of low back pain being developed. In the present study, we used the CCD model induced by inserting a stainless steel rod between vertebrae holes ( $L 4$ and/or L5) which has been proven to be a typical model that mimics the clinical etiology and various symptoms of low back pain ${ }^{3}$. In line with previous studies, we identified four different pain behaviors in the ipsilateral hindpaw of CCD rats, including mechanical hyperalgesia, thermal hyperalgesia, mechanical allodynia and cold allodynia [22, 29], which occurred as early as 1 day after surgery and maintained at least for 14 days.

Using CCD model, we found that the expression level of CXCR4 in compressed DRG dramatically increased revealed by immunofluorescent staining which was in accordance with a recent study [12]. The functional nature of the up-regulated CXCR4 was identified by our siRNA knockdown assay in which intraganglionar microinjection with CXCR4 siRNA obviously attenuated CCD-induced multiple pain behaviors. Additionally, blockade of CXCR4 with intervertebral foramen plerixafor injection showed the ability to reverse CCD-induced pain behaviors. All these results demonstrated that activation of CXCR4 in compressed DRG contributed to CCD-induced pain. As supporting, we previously have verified that intraganglionar microinjection with CXCR4 siRNA was able to relieve complete Freund's adjuvant (CFA)induced chronic inflammatory pain and spared nerve injury (SNI)-induced chronic neuropathic pain [26]. Moreover, the CXCR4 are found to be related with inflammatory pain-induced DRG neuronal hyperexcitability by our previous study in which plerixafor could inhibit the increased firing rate and reverse the lowered action potential rheobase [24]. Interestingly, it was reported that the DRG neuronal 
excitability extremely enhanced and the voltage-gated sodium currents in small and medium-sized DRG neurons also increased after compression [19, 21, 30]. It was well known that VGSCs were very important for electrogenesis and nerve impulse conduction, especially Nav1.8 which provided a significant contribution to action potential generation and Nav1.9 which contribute to setting the resting membrane potential and to neuronal excitability [31]. Here, we found for the first time that the expression of Nav1.8 and Nav1.9 in the compressed DRG neurons dramatically increased and the A-803467, a selective Nav1.8 blocker, as well as the local anesthetic ropivacine was efficient to alleviate CCD-induced pain behaviors after intervertebral foramen injection, which strongly supported that Nav1.8 and Nav1.9 were involved in the pathogenesis of low back pain. Our further study showed that intervertebral foramen plerixafor injection was sufficient to reducing the enhanced expression of Nav1.8 and Nav1.9 accompanying with easing pain behaviors, suggesting that the Nav1.8 and Nav1.9 were the downstream effector of CXCR4 activation for pain genesis. Actually, the contribution of CXCR4 activation-induced DRG neuronal protein synthesis to pain has been indicated in our previous study in which the protein translation inhibitor could block the acute mechanical hyperalgesia evoked by intraplantar injection of CXCL12, the ligand of CXCR4 [26]. In addition, we further have demonstrated that CXCL12-CXCR4 signaling could regulate the excitability of primary nociceptive neurons by up-regulating Nav1.8 expression and contribute to persistent pain and hyperalgesia induced by intraplantar bee venom injection [24]. Taking together, these findings evidenced that CXCR4-Nav1.8/Nav1.9 axis in compressed DRG was involved in CCD-induced low back pain. Recently, another study demonstrated that NaV1.6 and regulatory NaVß4 sodium channel in compressed DRG also play key roles in low back pain [22]. Besides, TRPV4, voltage-gated potassium current and hyperpolarization-activated cation current were all reported to be correlated with CCD-induced low back pain $[30,32,33]$. Whether these changed ion channels and currents shared the same upstream trigger mechanism (CXCR4 activation) remains unknown which are definitely needed to be explored in the future experiments.

\subsection{Blocking CXCR4 with plerixafor is a potential and efficient treatment for neuropathic pain}

Neuropathic pain is considered to be the most intractable chronic pain with current therapeutic approaches being unsatisfactory and inefficient. Thus, it is urgent to explore the new potential targets for pain management. CXCR4 is a seven transmembrane G protein-coupled receptor (GPCR) which has been widely concerned in the field of neuropathic pain research [9]. It is constitutively distributed in the somatosensory and nociceptive system [34]. In consistent with a recent study, we previously found that CXCR4 present in both non-peptidergic (IB4-positive) and peptidergic (SP-positive or CGRP-positive) primary nociceptor neurons and also co-localized with TRPV1, a thermonociceptor of primary sensory afferent [24]. In the central nervous system (CNS), spinal dorsal horn and thalamus as well as somatosensory cortex which are the primary centers for pain perception have a high level basal expression of CXCR4 [34]. All these findings strongly suggested that CXCR4 is closely related to pain. Here, we identified that the expression level of CXCR4 in normal DRG was at a low level, while it extremely enhanced under CCD state. In support of our finding, emerging evidence have demonstrated that CXCR4 expression in the nervous system was inducible under neuropathic pain state. In diabetic condition, the total number of DRG neurons expressing CXCR4 mRNA significantly increased comparing to normal DRG 
[35]. In chronic constriction injury (CCI) and SNI model, elevation of CXCR4 immunoreactivity has also been demonstrated in the DRG neuronal soma [36,37]. The expression level of CXCR4 in the CNS could also be obviously enhanced under central neuropathic pain situations such as central post stroke pain [10], spinal cord ischemia-reperfusion [38], spinal cord contusion injury [39]. This inducible expression pattern of CXCR4 enable CXCR4 as an ideal analgesic target. Actually, extensive behavioral pharmacology experiments have proven that blocking CXCR4 with its specific antagonist, plerixafor, was sufficient to prevent and reverse various neuropathic pain [9]. Importantly, plerixafor is hematopoietic stem cell mobilizer often used in clinic with its safety and efficacy has been approved by the U.S. Food and Drug Administration in 2008 [14]. It is noteworthy that intervertebral foramen plerixafor injection did not affect the basal pain response in normal rat, indicating that plerixafor could selectively relieve pathological pain without affecting the normal sensory or motor function. Additionally, we found that the analgesic duration of intervertebral foramen single plerixafor injection lasted at least for $24 \mathrm{~h}$ which was much longer than ropivacaine, a kind of local anesthetics commonly used for nerve blocking in clinic. We speculated that the protracted analgesic effect was largely because of the plerixafor not only reduced the expression of Nav1.8 and Nav1.9, but also has the ability to relieve inflammation in compressed DRG which was also a pivotal mechanism for neuronal hyperexcitability and chronic pain. In supporting, recent studies have demonstrated that the pro-inflammatory cytokines dramatically up-regulated accompanying with several anti-inflammatory cytokines down-regulated in compressed DRG [22], while plerixafor has been proven to be able to reducing the expression of TNF- $a$, IL-1 $\beta$, IL- 6 and inflammatory cell infiltration in the nervous system [10,35]. Collectively, the above characteristics and advantages of plerixafor make it an effective and applicable therapeutic medicine for neuropathic pain.

\subsection{Intervertebral foramen injection is an appliable treatment approach for low back pain}

As we known, many drugs used to treat neuropathic pain are delivered systematically and intrathecally which has a major strategic limitation. In addition to on-target effects, systemic delivery of these drugs has side effects because of off-target neural suppression in the peripheral nervous system (PNS), and even more so in the CNS. For plerixafor, the common adverse effects in clinical trials were dizziness, nausea and diarrhea which were observed in over $10 \%$ of patients, largely because CXCR4 is widely distributed in nervous system and presented in various kinds of cells [40]. An alternative approach for treating pain is delivering drugs into the DRG which has been recognized that DRG is a critical structure in sensory transduction and modulation, including pain transmission and the maintenance of persistent pain states. There are multiple changes in gene expression in DRG under pain condition, encompassing a large number of distinct family members including neuropeptides, receptors, ion channels, signal transduction molecules, synaptic vesicle proteins, and others [41, 42]. The functional consequence of these changes is sensitization and hyperexcitability of DRG neurons, which then leads to persistent pain [18]. Moreover, the DRG is consistently locating at the intervertebral neural foramina within a capsule which is an easily accessible target. All these unique characteristics make the DRG an ideal target for the treatment of chronic pain [43]. Clinically, the local anesthetics and steroid were usually injecting into the intervertebral foramina to block the electrogenesis and pain conduction at DRG level to cure various kinds of pain with the assistance of visual equipment, like the ultrasound and computed tomography [44]. In 
the present study, we provided preclinical evidence that intervertebral foramina injection at the compressed DRG of plerixafor or local anesthetics was sufficient to reverse the CCD-induced multiple pain behaviors. However, intervertebral foramina injection at the adjacent non-compressed DRG did not affect the pain behaviors, suggesting that the plerixafor dose for intervertebral foramina injection has no systemic effects. All of these data strongly indicated that intervertebral foramen plerixafor injection is an appliable treatment approach for low back pain.

\section{Conclusion}

The present study demonstrates for the first time that CXCR4 activation-mediated Nav1.8 and Nav1.9 upregulation in compressed DRG neurons was involved in CCD-induced low back pain and intervertebral foramina injection of plerixafor was an efficient and applicable strategy for the treatment of low back pain.

\section{Abbreviations}

CCD: chronic compression of the dorsal root ganglion; CCl: chronic constriction injury; CFA: complete Freund's adjuvant; CNS: central nervous system; CXCL12: chemokine CXC motif ligand 12; CXCR4: C chemokine receptor type 4, DRG: dorsal root ganglion; GPCR: G protein-coupled receptor; PNS: peripheral nervous system; PWMT: paw withdrawal mechanical threshold; PWTL: paw withdrawal thermal latency; SNI: spared nerve injury; VGSCs: voltage-gated sodium currents.

\section{Declarations}

\section{Competing interests}

The authors declare that they have no competing interests.

\section{Funding}

The funders had no role in study design, data collection and analysis, decision to publish, or preparation of the manuscript.

\section{Ethics approval and consent to participate}

This study followed the recommendations of the International Association for the Study of Pain (IASP) for the accomplishment of experimental studies in pain involving conscious animals. This study was also carried out in accordance with the recommendations of the Animal Care and Use Committee of Fuzhou General Hospital and performed in accordance with the updated Guide for the Care and Use of Laboratory Animals

\section{Acknowledgements}


This work was supported by the National Natural Science Foundation of China (NO. 81801101, N0.81870828, N0.81701091), the Natural Science Foundation of Fujian Province (NO.2019J05145) and the Outstanding Youth Project initiated by 900 Hospital (NO.2017Q1).

\section{Authors' contributions}

XZW and FY conceived and designed the experiments. FY, HHW and LC performed the experiments, XZW and FY analyzed and interpreted the data. GZC and XZW reviewed the manuscript. XZW and FY wrote the article. All authors listed, have made substantial, direct and intellectual contribution to the work, and approved it for publication.

\section{Availability of data and materials}

The datasets used and/or analysed during the current study are available from the corresponding author on reasonable request.

\section{Consent for publication}

The authors of this paper give consent to publication.

\section{References}

1. Hartvigsen J, Hancock MJ, Kongsted A, et al. What low back pain is and why we need to pay attention. LANCET. 2018;391(10137):2356-67.

2. Foster NE, Anema JR, Cherkin D, et al. Prevention and treatment of low back pain: evidence, challenges, and promising directions. LANCET. 2018;391(10137):2368-83.

3. Lin XY, Yang J, Li HM, Hu SJ, Xing JL. Dorsal root ganglion compression as an animal model of sciatica and low back pain. NEUROSCI BULL. 2012;28(5):618-30.

4. Wang T, Hurwitz O, Shimada SG, et al. Chronic Compression of the Dorsal Root Ganglion Enhances Mechanically Evoked Pain Behavior and the Activity of Cutaneous Nociceptors in Mice. PLOS ONE. 2015;10(9):e137512.

5. Han WJ, Chen L, Wang HB, et al. A Novel Nitronyl Nitroxide with Salicylic Acid Framework Attenuates Pain Hypersensitivity and Ectopic Neuronal Discharges in Radicular Low Back Pain. NEURAL PLAST. 2015;2015:752782.

6. Eckert F, Schilbach K, Klumpp L, et al. Potential Role of CXCR4 Targeting in the Context of Radiotherapy and Immunotherapy of Cancer. FRONT IMMUNOL. 2018;9:3018.

7. Kawaguchi N, Zhang TT, Nakanishi T. Involvement of CXCR4 in Normal and Abnormal Development. CELLS-BASEL. 2019;8(2).

8. Nagasawa T. CXCL12/SDF-1 and CXCR4. FRONT IMMUNOL. 2015;6:301.

9. Luo X, Wang X, Xia Z, Chung SK, Cheung CW. CXCL12/CXCR4 axis: an emerging neuromodulator in pathological pain. Rev Neurosci. 2016;27(1):83-92. 
10. Yang F, Luo WJ, Sun W, et al. SDF1-CXCR4 Signaling Maintains Central Post-Stroke Pain through Mediation of Glial-Neuronal Interactions. FRONT MOL NEUROSCI. 2017;10:226.

11. Li F, Xue ZY, Yuan Y, et al. Upregulation of CXCR4 through promoter demethylation contributes to inflammatory hyperalgesia in rats. CNS NEUROSCI THER. 2018;24(10):947-56.

12. Yu Y, Huang X, Di Y, Qu L, Fan N. Effect of CXCL12/CXCR4 signaling on neuropathic pain after chronic compression of dorsal root ganglion. Sci Rep. 2017;7(1):5707.

13. Wang J, Tannous BA, Poznansky MC, Chen H. CXCR4 antagonist AMD3100 (plerixafor): From an impurity to a therapeutic agent. PHARMACOL RES. 2020;159:105010.

14. Hummel S, Van Aken H, Zarbock A. Inhibitors of CXC chemokine receptor type 4: putative therapeutic approaches in inflammatory diseases. CURR OPIN HEMATOL. 2014;21(1):29-36.

15. Reaux-Le GA, Van Steenwinckel J, Rostene W, Melik PS. Current status of chemokines in the adult CNS. PROG NEUROBIOL. 2013;104:67-92.

16. Luo WJ, Yang F, Yang F, et al. Intervertebral Foramen Injection of Ozone Relieves Mechanical Allodynia and Enhances Analgesic Effect of Gabapentin in Animal Model of Neuropathic Pain. PAIN PHYSICIAN. 2017;20(5):E673-85.

17. Zhang ZJ, Guo JS, Li SS, et al. TLR8 and its endogenous ligand miR-21 contribute to neuropathic pain in murine DRG. J EXP MED. 2018;215(12):3019-37.

18. Esposito MF, Malayil R, Hanes M, Deer T. Unique Characteristics of the Dorsal Root Ganglion as a Target for Neuromodulation. PAIN MED. 2019;20(Suppl 1):S23-30.

19. Fan N, Donnelly DF, LaMotte RH. Chronic compression of mouse dorsal root ganglion alters voltagegated sodium and potassium currents in medium-sized dorsal root ganglion neurons. $J$ NEUROPHYSIOL. 2011;106(6):3067-72.

20. Song Y, Li HM, Xie RG, et al. Evoked bursting in injured Abeta dorsal root ganglion neurons: a mechanism underlying tactile allodynia. PAIN. 2012;153(3):657-65.

21. Fan N, Sikand P, Donnelly DF, Ma C, Lamotte RH. Increased Na+ and K+ currents in small mouse dorsal root ganglion neurons after ganglion compression. J NEUROPHYSIOL. 2011;106(1):211-8.

22. Xie W, Zhang J, Strong JA, Zhang JM. Role of NaV1.6 and NaVbeta4 Sodium Channel Subunits in a Rat Model of Low Back Pain Induced by Compression of the Dorsal Root Ganglia. NEUROSCIENCE. 2019;402:51-65.

23. Huang J, Vanoye CG, Cutts A, et al. Sodium channel NaV1.9 mutations associated with insensitivity to pain dampen neuronal excitability. J CLIN INVEST. 2017;127(7):2805-14.

24. Yang F, Sun W, Yang Y, et al. SDF1-CXCR4 signaling contributes to persistent pain and hypersensitivity via regulating excitability of primary nociceptive neurons: involvement of ERKdependent Nav1.8 up-regulation. J Neuroinflammation. 2015;12:219.

25. Watanabe K, Yabuki S, Sekiguchi M, Kikuchi S, Konno S. Etanercept attenuates pain-related behavior following compression of the dorsal root ganglion in the rat. EUR SPINE J. 2011;20(11):1877-84. 
26. Yang F, Sun W, Luo WJ, et al. SDF1-CXCR4 Signaling Contributes to the Transition from Acute to Chronic Pain State. MOL NEUROBIOL. 2017;54(4):2763-75.

27. Qiu F, Li Y, Fu Q, et al. Stromal Cell-Derived Factor 1 Increases Tetrodotoxin-Resistant Sodium Currents Nav1.8 and Nav1.9 in Rat Dorsal Root Ganglion Neurons via Different Mechanisms. NEUROCHEM RES. 2016;41(7):1587-603.

28. Vlaeyen J, Maher CG, Wiech K, et al. Low back pain. NAT REV DIS PRIMERS. 2018;4(1):52.

29. Liang $L$, Zhang J, Tian $L$, et al. AXL signaling in primary sensory neurons contributes to chronic compression of dorsal root ganglion-induced neuropathic pain in rats. MOL PAIN. 2020;16:2069262706.

30. Zhang M, Han W, Zheng J, et al. Inhibition of Hyperpolarization-Activated Cation Current in MediumSized DRG Neurons Contributed to the Antiallodynic Effect of Methylcobalamin in the Rat of a Chronic Compression of the DRG. NEURAL PLAST. 2015;2015:197392.

31. Theriault $\mathrm{O}$, Chahine M. Correlation of the electrophysiological profiles and sodium channel transcripts of individual rat dorsal root ganglia neurons. FRONT CELL NEUROSCI. 2014;8:285.

32. Qu YJ, Zhang X, Fan ZZ, et al. Effect of TRPV4-p38 MAPK Pathway on Neuropathic Pain in Rats with Chronic Compression of the Dorsal Root Ganglion. BIOMED RES INT. 2016;2016:6978923.

33. Ma C, Rosenzweig J, Zhang P, Johns DC, LaMotte RH. Expression of inwardly rectifying potassium channels by an inducible adenoviral vector reduced the neuronal hyperexcitability and hyperalgesia produced by chronic compression of the spinal ganglion. MOL PAIN. 2010;6:65.

34. Reaux-Le GA, Rivat C, Kitabgi P, PohI M, Melik PS. Cellular and subcellular localization of CXCL12 and CXCR4 in rat nociceptive structures: physiological relevance. EUR J NEUROSCI. 2012;36(5):261931.

35. Menichella DM, Abdelhak B, Ren D, Shum A, Frietag C, Miller RJ. CXCR4 chemokine receptor signaling mediates pain in diabetic neuropathy. MOL PAIN. 2014;10:42.

36. Dubovy P, Klusakova I, Svizenska I, Brazda V. Spatio-temporal changes of SDF1 and its CXCR4 receptor in the dorsal root ganglia following unilateral sciatic nerve injury as a model of neuropathic pain. HISTOCHEM CELL BIOL. 2010;133(3):323-37.

37. Bai L, Wang X, Li Z, et al. Upregulation of Chemokine CXCL12 in the Dorsal Root Ganglia and Spinal Cord Contributes to the Development and Maintenance of Neuropathic Pain Following Spared Nerve Injury in Rats. NEUROSCI BULL. 2016;32(1):27-40.

38. Li XQ, Zhang ZL, Tan WF, Sun XJ, Ma H. Down-Regulation of CXCL12/CXCR4 Expression Alleviates Ischemia-Reperfusion-Induced Inflammatory Pain via Inhibiting Glial TLR4 Activation in the Spinal Cord. PLOS ONE. 2016;11(10):e163807.

39. Knerlich-Lukoschus F, von der Ropp-Brenner B, Lucius R, Mehdorn HM, Held-Feindt J. Spatiotemporal CCR1, CCL3(MIP-1alpha), CXCR4, CXCL12(SDF-1alpha) expression patterns in a rat spinal cord injury model of posttraumatic neuropathic pain. J Neurosurg Spine. 2011;14(5):583-97.

40. Wagstaff AJ. Plerixafor: in patients with non-Hodgkin's lymphoma or multiple myeloma. DRUGS. 2009;69(3):319-26. 
41. Liem L, van Dongen E, Huygen FJ, Staats P, Kramer J. The Dorsal Root Ganglion as a Therapeutic Target for Chronic Pain. Reg Anesth Pain Med. 2016;41(4):511-9.

42. Krames ES. The role of the dorsal root ganglion in the development of neuropathic pain. PAIN MED. 2014;15(10):1669-85.

43. Ahimsadasan N, Reddy V, Kumar A. Neuroanatomy, Dorsal Root Ganglion. 2020.

44. Qin DA, Song JF, Song LP, Feng GS. Integrated approach to pain management for a patient with multiple bone metastases of uterine cervical cancer. J INT MED RES. 2018;46(5):2023-30.

\section{Figures}

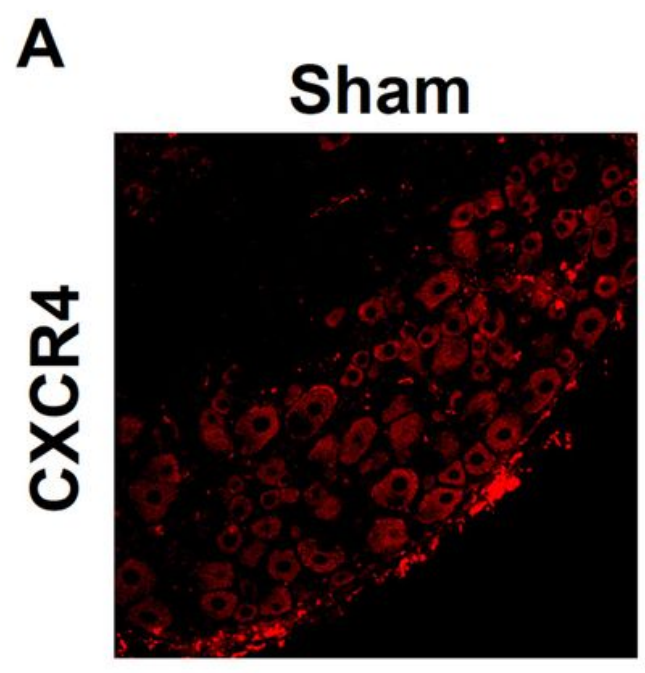

CCD

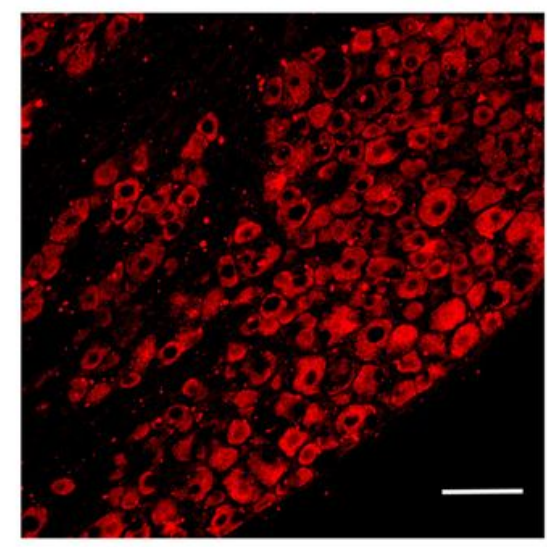

CXCR4

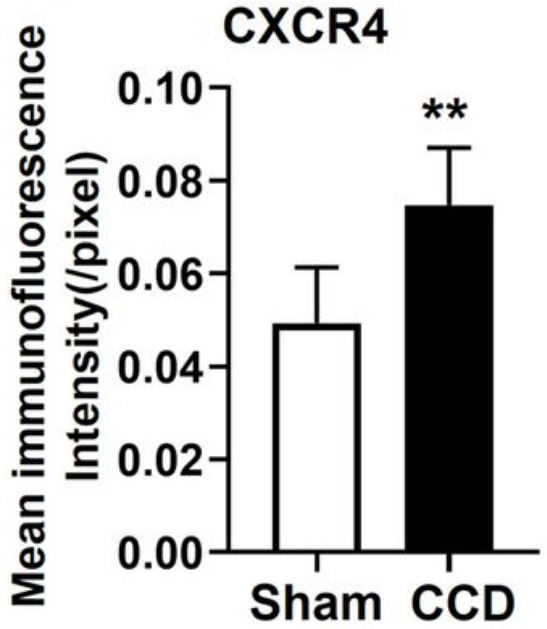

Figure 1

Up-regulation of CXCR4 protein level in the rat compressed DRG. A Representative immunofluorescence photomicrographs of CXCR4 in lumbar DRG from sham and CCD rat on postoperative day 3. Scale bar: $100 \mu \mathrm{m}$. B Quantification of the mean immunofluorescence intensity of CXCR4 showing an increase in CXCR4 expression following CCD ( $n=6$ per group) ${ }^{\star} P<0.005, C C D$ vs. Sham. CCD, chronic compression of dorsal root ganglion. 

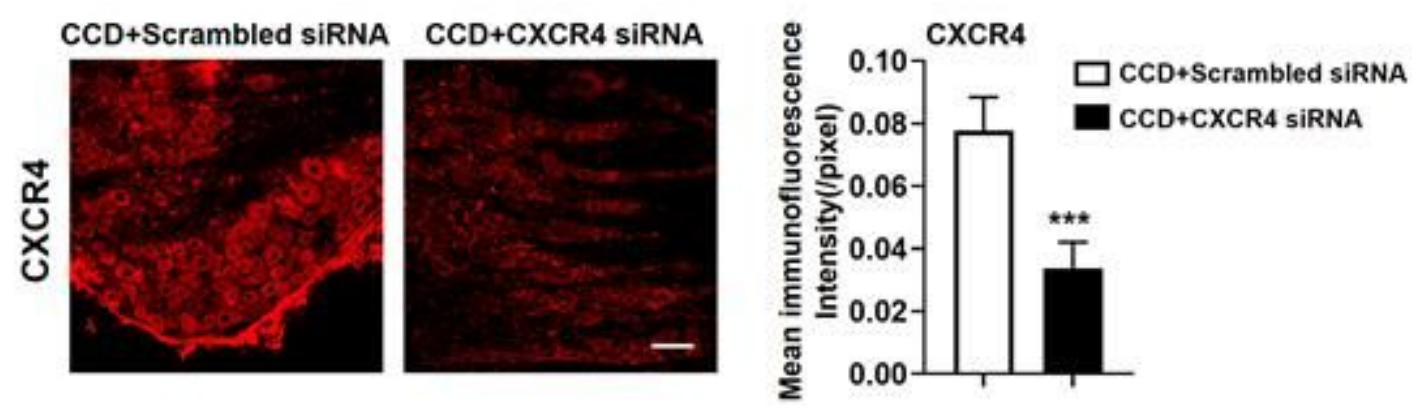

C

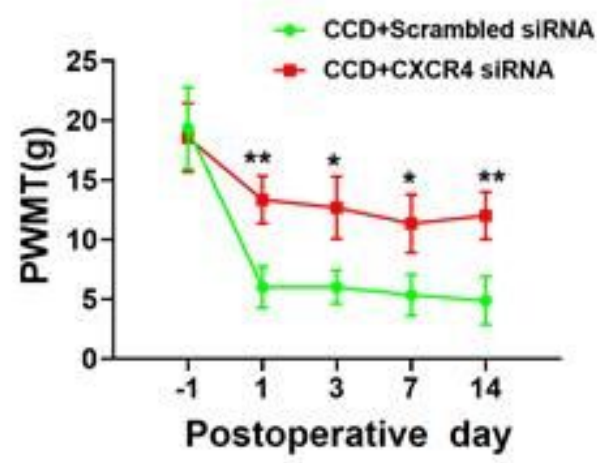

E Mechanical allodynia test

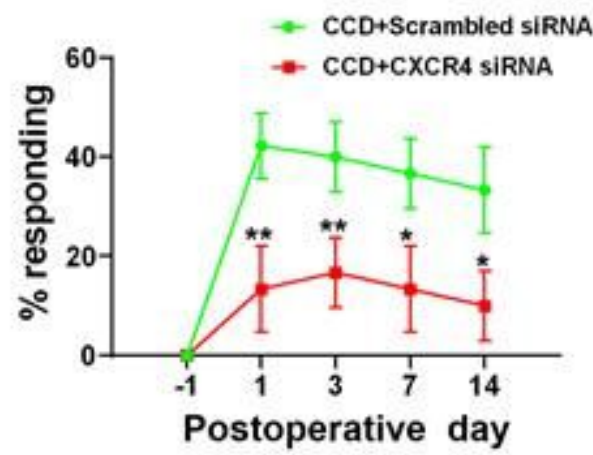

\section{Thermal stimulation test}

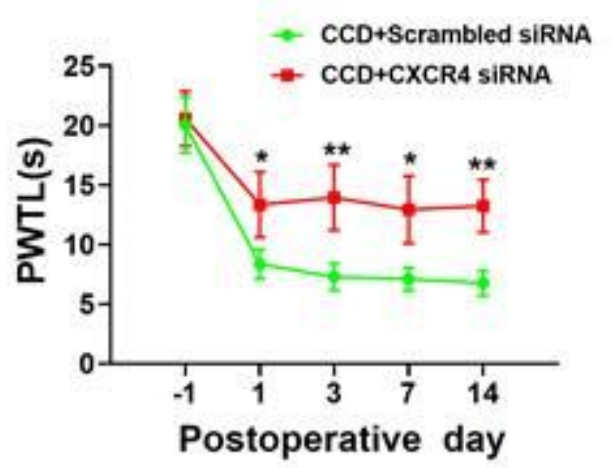

\section{F Cold allodynia test}

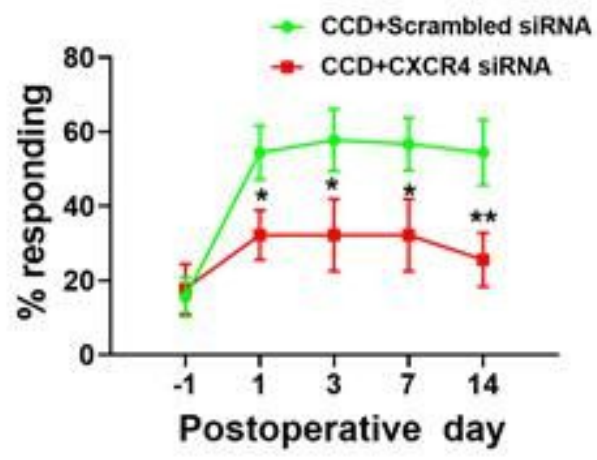

Figure 2

Knockdown of CXCR4 reduces pain behaviors in CCD rats. A Representative immunofluorescence photomicrographs of CXCR4 in lumbar DRG from CCD rat after injection of either CXCR4 siRNA or scrambled siRNA. Scale bar: $100 \mu \mathrm{m}$. B Quantification of the mean immunofluorescence intensity of CXCR4 showing down-regulation of CXCR4 expression following CXCR4 siRNA injection ( $n=6$ per group) ${ }^{\star} * * \mathrm{P}<0.001 \mathrm{CCD}+\mathrm{CXCR} 4$ siRNA vs. CCD + Scrambled siRNA. C Time course changes of PWMT in CCD rats after injection of either CXCR4 siRNA or scrambled siRNA. D Time course changes of PWTL in CCD rats after injection of either CXCR4 siRNA or scrambled siRNA. E Time course effect of CXCR4 siRNA injection on CCD-induced mechanical allodynia. F Time course effect of CXCR4 siRNA injection on CCDinduced cold allodynia. ${ }^{*} \mathrm{P}<0.05,{ }^{*} \mathrm{P}<0.005, \mathrm{CCD}+\mathrm{CXCR} 4$ siRNA vs. CCD + Scrambled siRNA $(\mathrm{n}=9$ per 
group).CCD, chronic compression of dorsal root ganglion; PWMT, paw withdrawal mechanical threshold; PWTL, paw withdrawal thermal latency.

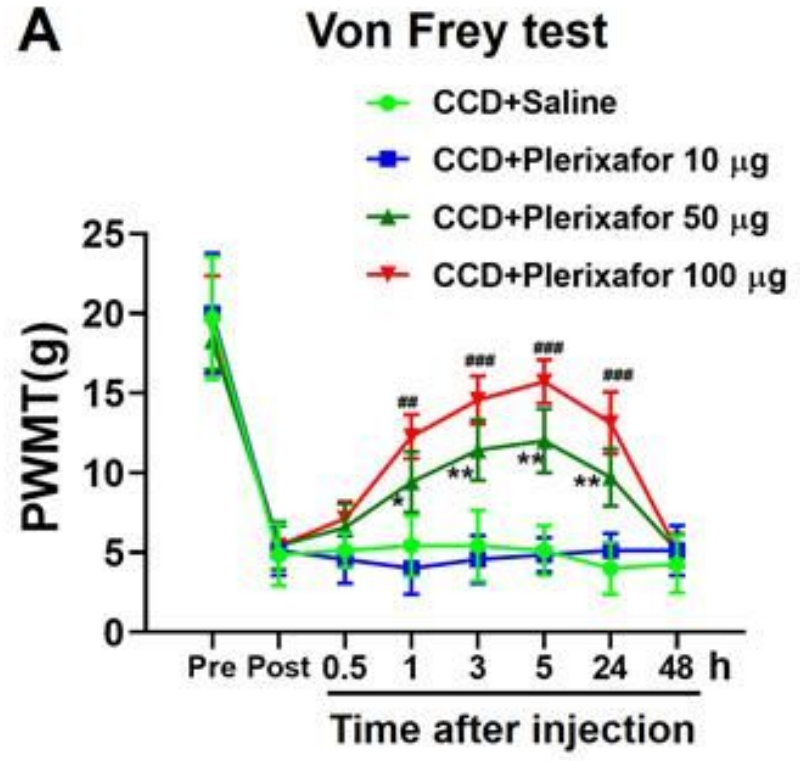

C Mechanical allodynia test

$\rightarrow \mathrm{CCD}+$ Saline

- CCD+Plerixafor $10 \mu \mathrm{g}$

- CCD+Plerixafor $50 \mu \mathrm{g}$

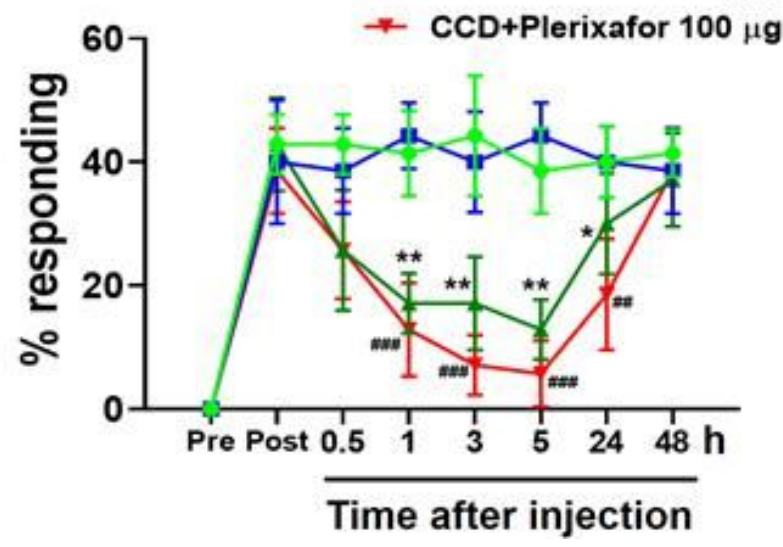

B Thermal stimulation test

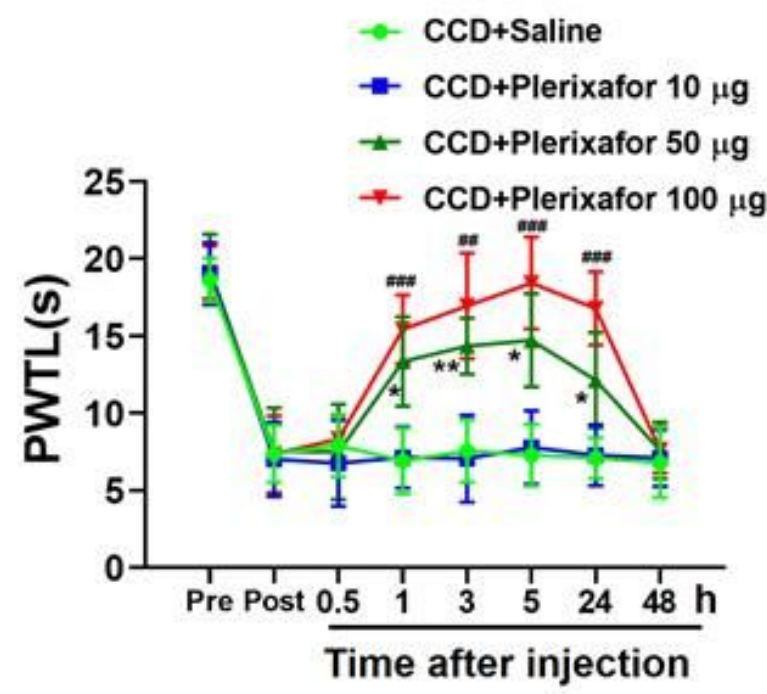

D Cold allodynia test

- CCD+Saline

- CCD+Plerixafor $10 \mu \mathrm{g}$

$\$$ - CCD+Plerixafor $50 \mu \mathrm{g}$

* CCD+Plerixafor $100 \mu \mathrm{g}$

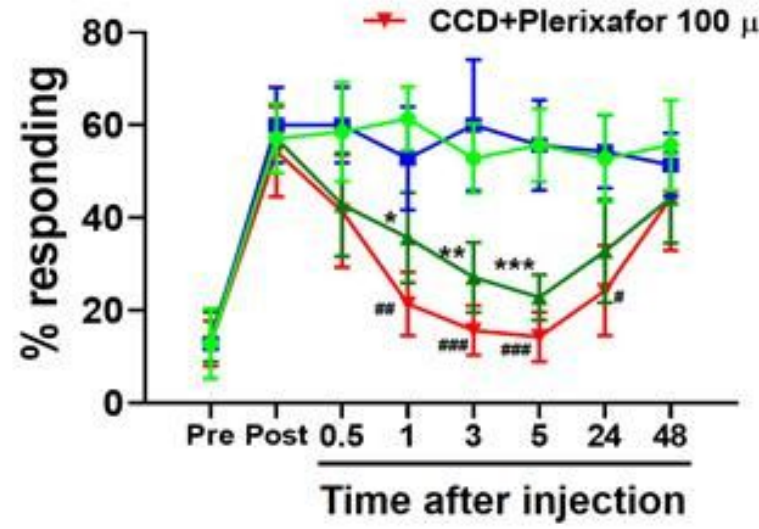

Figure 3

Intervertebral foramen plerixafor injection at $L 5$ level reduces pain behaviors in CCD rats. A Time course changes of PWMT in CCD rats after intervertebral foramen injection of plerixafor. B Time course changes of PWTL in CCD rats after after intervertebral foramen injection of plerixafor. C Time course effect of intervertebral foramen plerixafor injection on CCD-induced mechanical allodynia. D Time course effect of intervertebral foramen plerixafor injection on CCD-induced cold allodynia. ${ }^{*} P<0.05, * * P<0.005$,

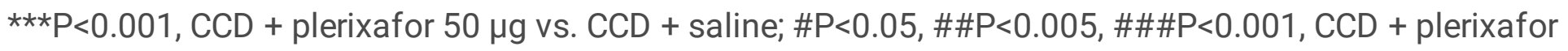
$100 \mu \mathrm{g}$ vs. $C C D+$ saline ( $n=7$ per group). $C C D$, chronic compression of dorsal root ganglion; PWMT, paw withdrawal mechanical threshold; PWTL, paw withdrawal thermal latency. 
A

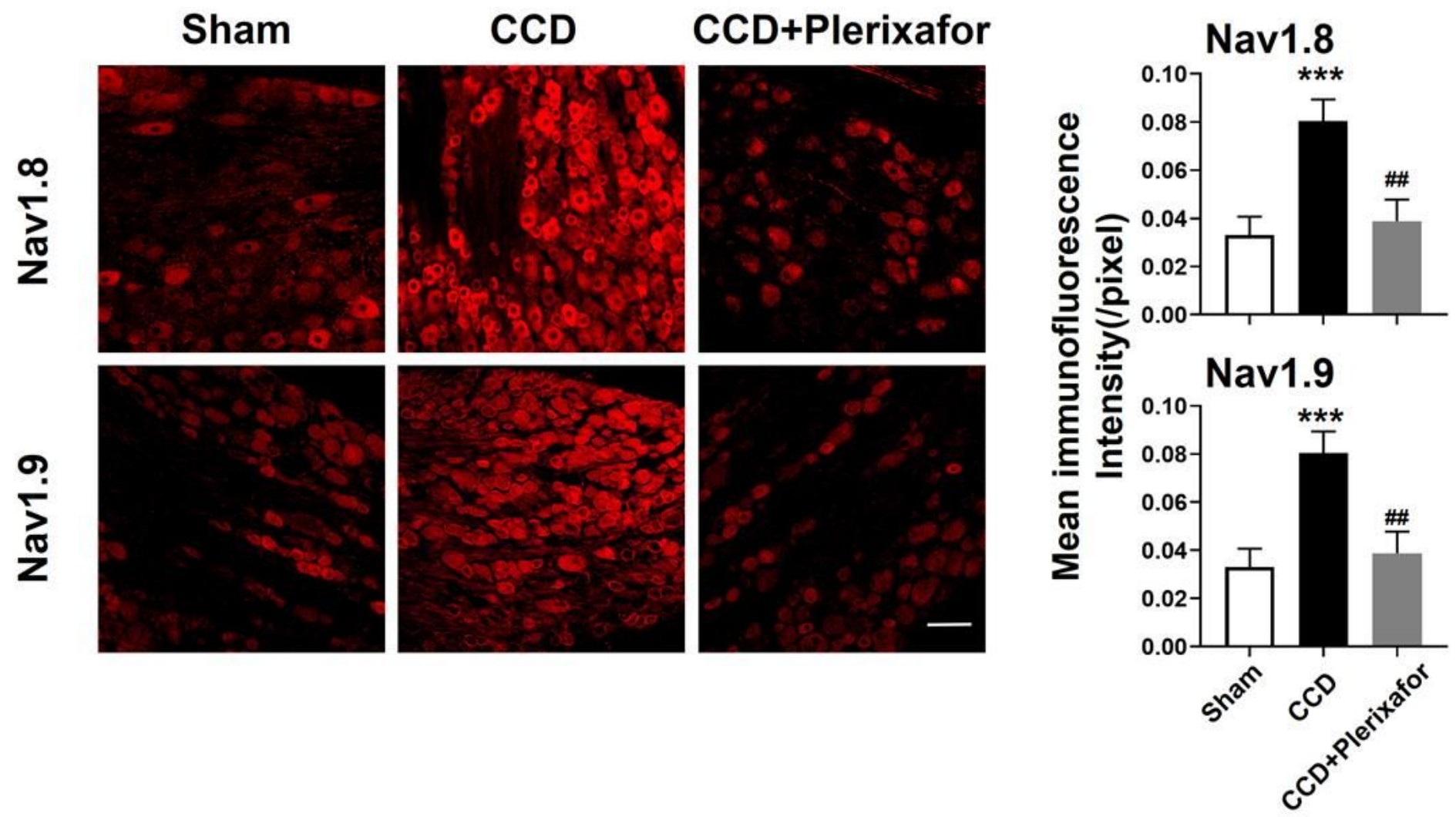

Figure 4

Intervertebral foramen plerixafor injection attenuates CCD-induced up-regulation of Nav1.8 and Nav1.9 in compressed DRG. A Representative immunofluorescence photomicrographs of Nav1.8 and Nav1.9 in L5 DRG from sham, CCD and CCD receiving plerixafor injection rats. Scale bar: $100 \mu \mathrm{m}$. B Quantification of the mean immunofluorescence intensity of Nav1.8 and Nav1.9 in L5 DRG showing intervertebral foramen plerixafor injection decreases CCD-induced high expression of Nav1.8 and Nav1.9 ( $n=6$ per group).

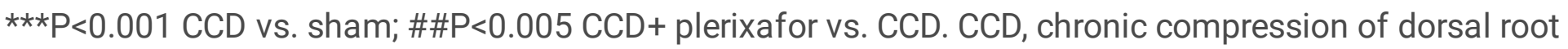
ganglion. 

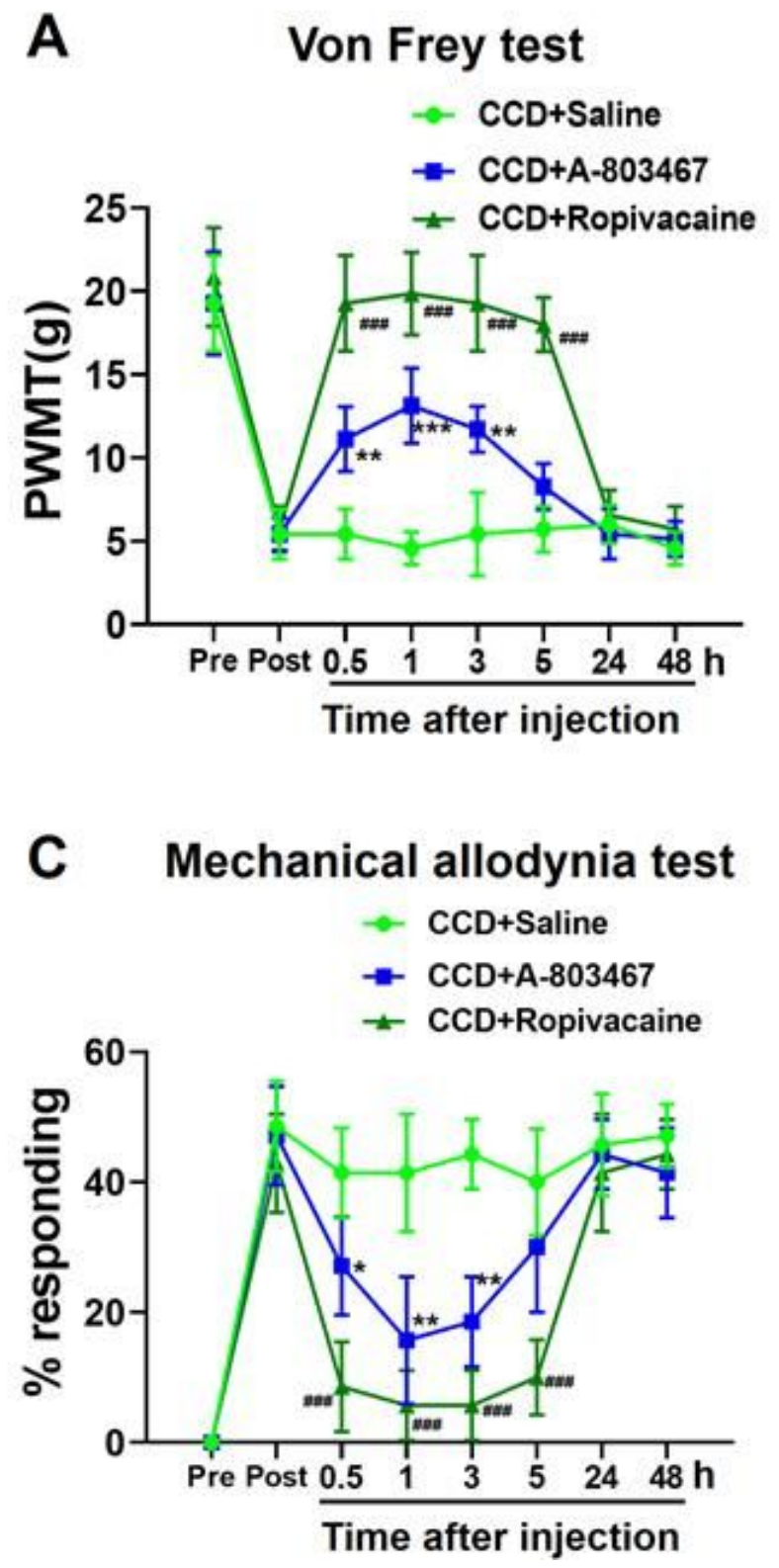
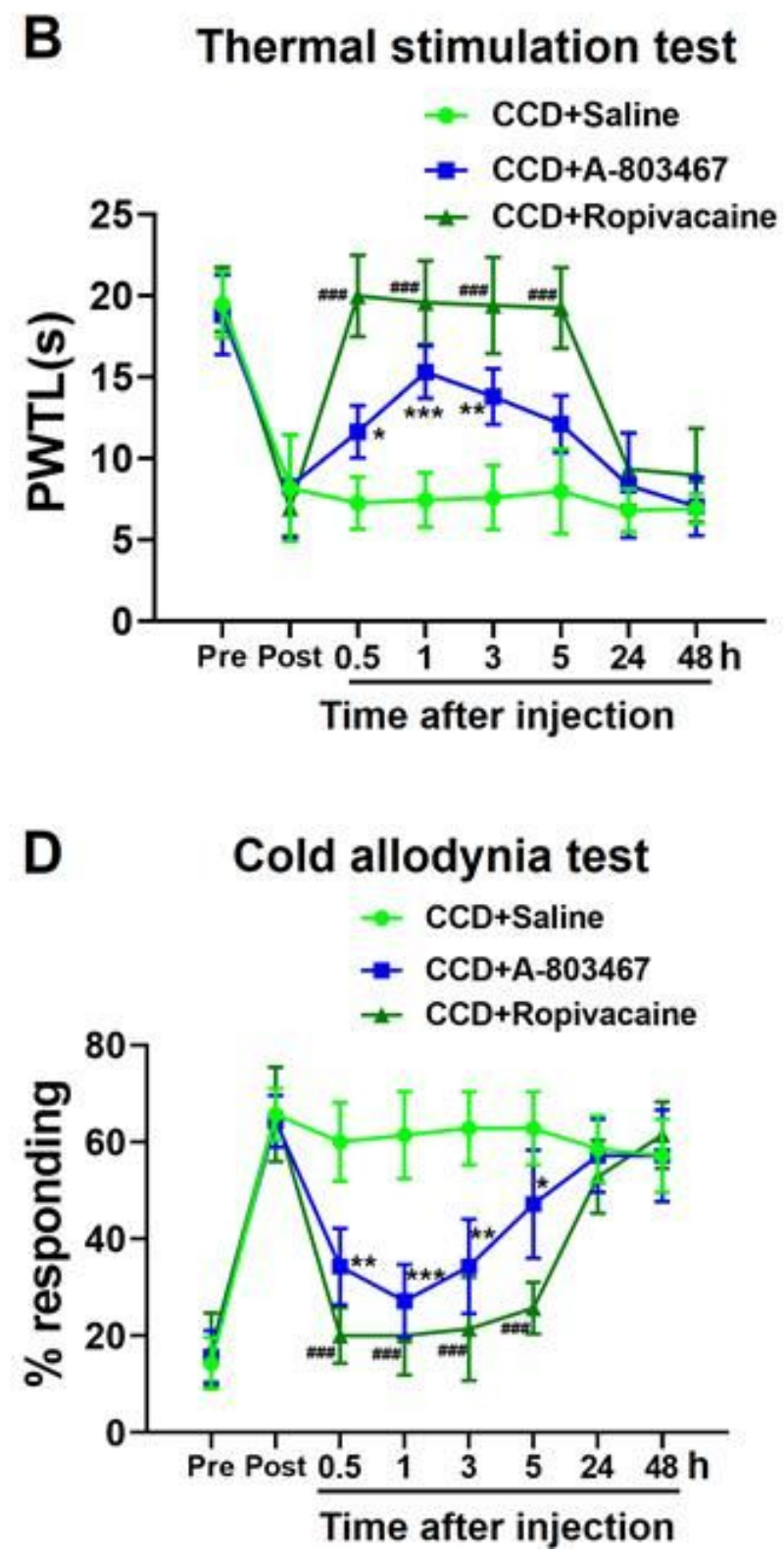

Figure 5

Intervertebral foramen injection of A-803467 or ropivacaine reduce pain behaviors in CCD rats. A Time course changes of PWMT in CCD rats after intervertebral foramen injection of A-803467 or ropivacaine at L5 level. B Time course changes of PWTL in CCD rats after intervertebral foramen injection of A-803467 or ropivacaine at $L 5$ level. C Time course effect of intervertebral foramen A-803467 or ropivacaine injection at $L 5$ level on CCD-induced mechanical allodynia. D Time course effect of intervertebral foramen A-803467 or ropivacaine injection at $L 5$ level on CCD-induced cold allodynia. ${ }^{*} P<0.05,{ }^{*} P<0.005$, $\star * * P<0.001, C C D+A-803467$ vs. CCD + saline; \#\#\#P<0.001, CCD + ropivacaine vs. $C C D+$ saline $(n=7$ per group). CCD, chronic compression of dorsal root ganglion; PWMT, paw withdrawal mechanical threshold; PWTL, paw withdrawal thermal latency. 

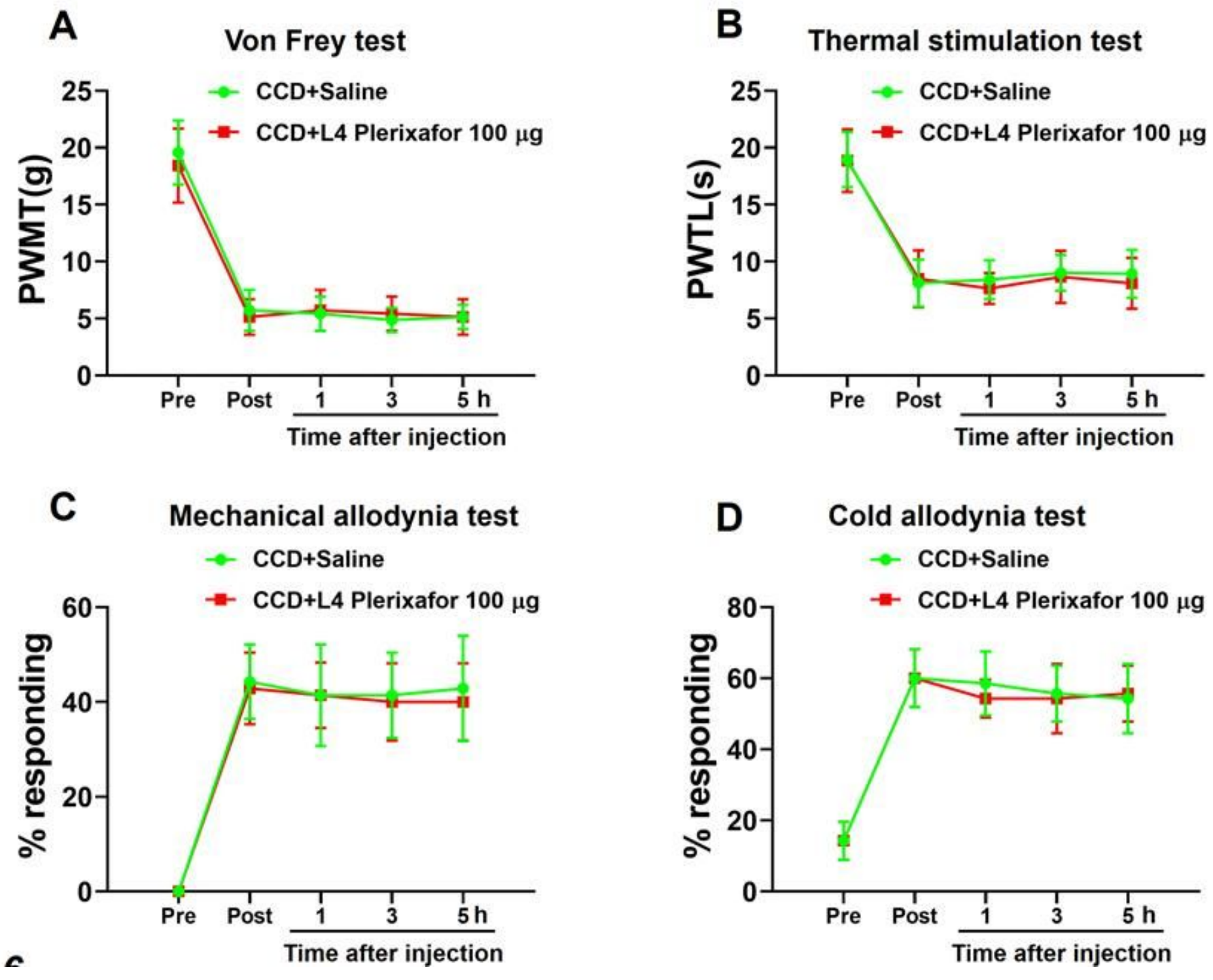

Figure 6

Intervertebral foramen plerixafor injection at L4 level has no effect on CCD-induced pain behaviors. A Time course changes of PWMT in CCD rats after intervertebral foramen injection of plerixafor at L4 level. B Time course changes of PWTL in CCD rats after intervertebral foramen injection of plerixafor at L4 level. C Time course effect of intervertebral foramen plerixafor injection at L4 level on CCD-induced mechanical allodynia. D Time course effect of intervertebral foramen plerixafor injection at L4 level on CCD-induced cold allodynia ( $n=7$ per group). CCD, chronic compression of dorsal root ganglion; PWMT, paw withdrawal mechanical threshold; PWTL, paw withdrawal thermal latency. 
A

\section{Von Frey test}

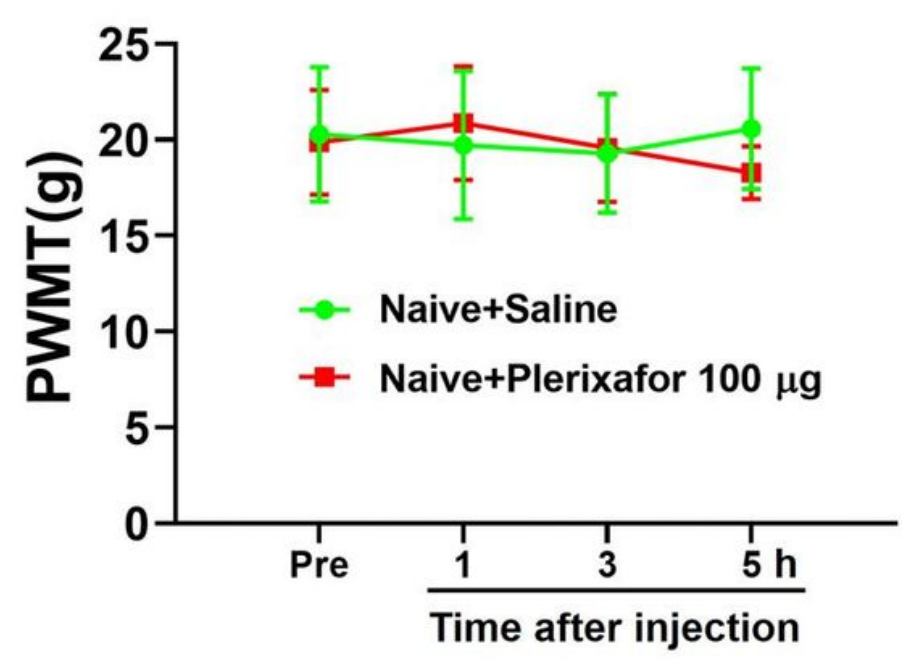

B

\section{Thermal stimulation test}

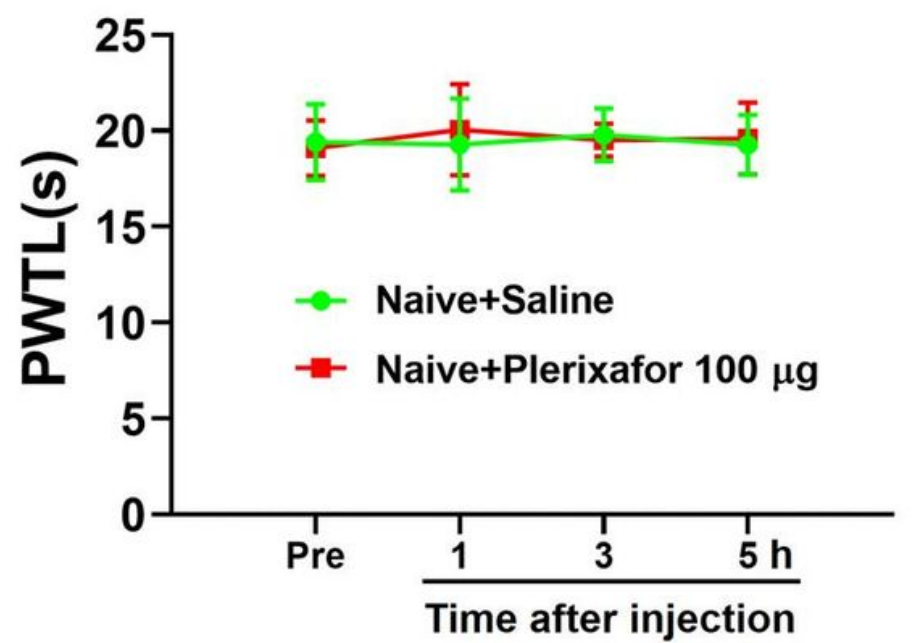

Figure 7

Intervertebral foramen plerixafor injection has no effect on baseline values of PWMT and PWTL in normal rats. A Time course changes of PWMT in normal rats after intervertebral foramen injection of plerixafor. B Time course changes of PWTL in normal rats after intervertebral foramen injection of plerixafor ( $n=7$ per group). PWMT, paw withdrawal mechanical threshold; PWTL, paw withdrawal thermal latency. 